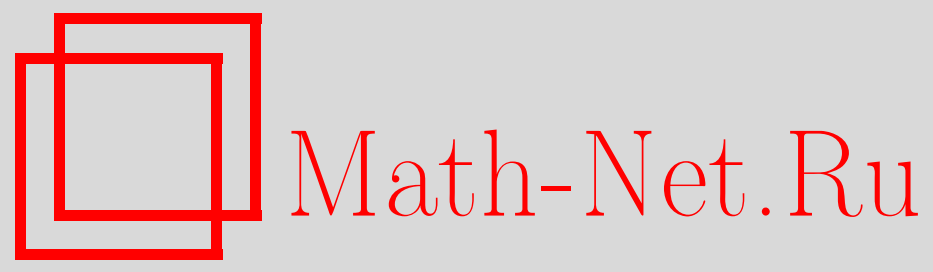

К. Н. Панков, Верхняя граница для числа функций, удовлетворяющих строгому лавинному критерию, Дискрет. матем., 2005, том 17, выпуск 2, 95-101

DOI: https://doi.org/10.4213/dm101

Использование Общероссийского математического портала Math-Net.Ru подразумевает, что вы прочитали и согласны с пользовательским соглашением http: //www . mathnet.ru/rus/agreement

Параметры загрузки:

IP : 3.80 .181 .102

26 апреля 2023 г., 16:54:12 
Удк 519.7

\title{
Верхняя граница для числа функций, удовлетворяющих строгому лавинному критерию
}

\author{
(c) 2005 г. $\quad$ K. Н. Панков
}

\begin{abstract}
Строгий лавинный критерий был предложен при изучении критериев построения некоторых криптографических функций. Двоичная функция $f(x), x \in V_{n}$, удовлетворяет этому критерию, если при замене любой координаты вектора $x$ ее дополнением значение $f(x)$ изменяется ровно в половине случаев. В данной работе представлена верхняя граница для числа таких функций при достаточно больших $n$.
\end{abstract}

Пусть $V_{n}$ - множество двоичных векторов размерности $n$. Двоичная функция $f(x)$, $x \in V_{n}$, удовлетворяет строгому лавинному критерию, если при замене любой координаты вектора $x$ дополнением значение $f(x)$ изменяется ровно в половине случаев. Это эквивалентно тому, что для любого $i=1,2, \ldots$

$$
\left\|f(x) \oplus f\left(x \oplus e_{i}\right)\right\|=\sum_{\beta \in V_{n}}\left(f(\beta) \oplus f\left(\beta \oplus e_{i}\right)\right)=2^{n-1},
$$

где $e_{i} \in V_{n}$ - вектор веса 1 с единицей на $i$-м месте.

Данный критерий был введен в [1]. Как и для любого другого критерия, для строгого лавинного критерия представляет интерес подсчет числа удовлетворяющих ему функцй от $n$ переменных для произвольного $n$. Данное число обозначим через $S^{n}$. К настоящему времени получены лишь оценки этого числа. Нижние оценки, представленные в $[2,3,4]$ определяются путем построения классов функций, удовлетворяющих изучаемому критерию, и вычисления мощностей этих классов. В [5] с помощью представления элементов $V_{n}$ как вершин $n$-мерного гиперкуба доказана самая сильная на нынешний момент нижняя оценка для $S^{n}$, справедливая для достаточно больших $n$ :

$$
S^{n} \geqslant \frac{\exp _{2}\left(2^{n}-n^{2} / 2+n\right)}{\pi^{n / 2}},
$$

где $\exp _{2}(x)=2^{x}$.

Нетривиальная верхняя граница для $S^{n}$ была построена в [6].

Пусть на множестве $V_{n}$ задано равномерное распределение вероятностей, тогда $f(x)$ можно рассматривать как случайную величину. Будем говорить, что двоичная функция $f(x), x=\left(x_{1}, \ldots, x_{n}\right) \in V_{n}$, зависит на $50 \%$ от входа $x_{i}$, если вероятность того, что она принимает разные значения на векторах, различающихся только $i$-й координатой, равна $1 / 2$. 
Обозначим через $S(n, k)$ число функций от $n$ переменных, зависящих на $50 \%$ от переменных $x_{1}, \ldots, x_{k}$. Функция $f(x)$ зависит на $50 \%$ от переменных $x_{1}, \ldots, x_{k}$ тогда и только тогда, когда

$$
\sum_{\beta \in V_{n}}\left(\begin{array}{c}
f(\beta) \oplus f\left(\beta \oplus e_{1}\right) \\
\ldots \\
f(\beta) \oplus f\left(\beta \oplus e_{k}\right)
\end{array}\right)=\left(\begin{array}{c}
2^{n-1} \\
\ldots \\
2^{n-1}
\end{array}\right)
$$

При этом $S^{n}=S(n, n)$.

В [6] найдены точные значения для $S(n, 1)$ и $S(n, 2)$ :

$$
\begin{aligned}
& S(n, 1)=\left(\begin{array}{c}
2^{n-1} \\
2^{n-2}
\end{array}\right) 2^{n-1}, \\
& S(n, 2)=\sum_{i=0}^{2^{n-3}}\left(\begin{array}{c}
2^{n-2} \\
2 i
\end{array}\right) 8^{2^{n-2}-2 i} 2^{2 i} \sum_{j=0}^{i}\left(\begin{array}{c}
2 i \\
2 j
\end{array}\right)\left(\begin{array}{c}
2 j \\
j
\end{array}\right)\left(\begin{array}{c}
2 i-2 j \\
i-j
\end{array}\right) .
\end{aligned}
$$

Очевидно, что $S^{n} \leqslant S(n, k)$ для любого $k \leqslant n$, однако вычисление точных значений $S(n, k)$ при $k \geqslant 2$ представляет значительную сложность.

Обозначим через $A_{i}$ событие, заключающееся в том, что случайная двоичная функция не является зависимой на $50 \%$ от переменной $x_{i}$. Тогда

$$
\begin{aligned}
\mathbf{P}\left(A_{i}\right) & =1-\frac{S(n, 1)}{\exp _{2}\left(2^{n}\right)}<1, \\
\mathbf{P}\left(A_{i} \cap A_{j}\right) & =1-\frac{S(n, 2)}{\exp _{2}\left(2^{n}\right)}<1, \quad i \neq j .
\end{aligned}
$$

В [6] предложена следующая верхняя оценка для числа функщий, удовлетворяющих строгому лавинному критерию:

$$
S^{n} \leqslant 2^{2^{n}}-\frac{2^{2^{n}} n^{2}\left(\mathrm{P}\left(A_{1}\right)\right)^{2}}{n \mathrm{P}\left(A_{1}\right)+n(n-1) \mathrm{P}\left(A_{1} \cap A_{2}\right)}
$$

В данной работе доказывается асимптотическая формула для $S(n, k) / \exp _{2}\left(2^{n}\right)$ в случае произвольного натурального фиксированного $k$ и из нее в качестве следствия выводится верхняя оценка $S^{n}$, улучшающая оценку (1).

Теорема 1. Пусть $n \rightarrow \infty, k-$ фиксированное натуральное число. Тогда

$$
\frac{S(n, k)}{\exp _{2}\left(2^{n}\right)}=2^{2 k-1} \pi^{-k / 2} 2^{-n k / 2}+o\left(2^{-n(k+1) / 2}\right) .
$$

Доказательство. Рассмотрим сумму

$$
\Sigma_{n}=\sum_{\beta \in V_{n}}\left(\begin{array}{c}
f(\beta) \oplus f\left(\beta \oplus e_{1}\right) \\
\cdots \\
f(\beta) \oplus f\left(\beta \oplus e_{k}\right)
\end{array}\right)
$$

и разобьем область суммирования на $2^{n-k}$ областей, соответствующих векторам $\beta$ с оди- 
наковыми последними $n-k$ координатами, набор которых будем обозначать $\alpha$. Тогда

$$
\begin{aligned}
& \Sigma_{n}=\sum_{\alpha \in V_{n-k}}\left(\sum_{\gamma \in V_{k}}\left(\begin{array}{c}
f(\gamma \alpha) \oplus f\left(\gamma \alpha \oplus e_{1}\right) \\
\ldots \\
f(\gamma \alpha) \oplus f\left(\gamma \alpha \oplus e_{k}\right)
\end{array}\right)\right) \\
& =\sum_{\alpha \in V_{n-k}} 2\left(\begin{array}{c}
\sum_{t \in V_{k-1}}(f(1 t \alpha) \oplus f(0 t \alpha)) \\
\ldots \\
\sum_{t \in V_{k-1}}\left(f\left(t_{1} \ldots t_{i-1} 1 t_{i} \ldots t_{k} \alpha\right) \oplus f\left(t_{1} \ldots t_{i-1} 0 t_{i} \ldots t_{k-1} \alpha\right)\right) \\
\ldots \\
\left.\sum_{t \in V_{k-1}}(f(t) \alpha) \oplus f(t 0 \alpha)\right)
\end{array}\right) \text {, }
\end{aligned}
$$

здесь $t=\left(t_{1}, \ldots, t_{k-1}\right) \in V_{k-1},(1 t \alpha)$ означает конкатенацию векторов $1, t$ и $\alpha, \sum$ означает сложение в поле действительных чисел, а $\oplus$ - сложение по модулю 2.

Пусть функция $f$ выбирается случайно и равновероятно из множества $B_{n}$ всех двоичных функций от $n$ переменных. Это эквивалентно независимому равновероятному выбору значений $f(\beta)$ из $\{0,1\}$ для всех $\beta$ из множества $V_{n}$. Очевидно, что

$$
\mathbf{P}\left(\sum_{\beta \in V_{n}}\left(\begin{array}{c}
f(\beta) \oplus f\left(\beta \oplus e_{1}\right) \\
\cdots \\
f(\beta) \oplus f\left(\beta \oplus e_{k}\right)
\end{array}\right)=\left(\begin{array}{c}
2^{n-1} \\
\ldots \\
2^{n-1}
\end{array}\right)\right)=\frac{S(n, k)}{2^{2^{n}}}
$$

Введем случайный вектор

$$
\eta_{\alpha}=\left(\begin{array}{c}
\eta_{\alpha}^{1} \\
\cdots \\
\eta_{\alpha}^{k}
\end{array}\right)=\left(\begin{array}{c}
\sum_{t \in V_{k-1}}(f(1 t \alpha) \oplus f(0 t \alpha)) \\
\ldots \\
\sum_{t \in V_{k-1}}(f(t 1 \alpha) \oplus f(t 0 \alpha))
\end{array}\right)
$$

Слагаемые в каждой компоненте вектора $\eta_{\alpha}$ независимы и принимают значения 0 и 1 с вероятностью 1/2. Поэтому каждая компонента вектора $\eta_{\alpha}$ имеет биномиальное распределение с параметрами $\left(2^{k-1}, 1 / 2\right)$.

Очевидно, что все случайные векторы $\eta_{\alpha}$ независимы, одинаково распределены и

$$
\begin{gathered}
\mathbf{M} \eta_{\alpha}=\left(\begin{array}{c}
2^{k-2} \\
\ldots \\
2^{k-2}
\end{array}\right), \\
\mathbf{P}\left(\sum_{\beta \in V_{n}}\left(\begin{array}{c}
f(\beta) \oplus f\left(\beta \oplus e_{1}\right) \\
f(\beta) \oplus f\left(\beta \oplus e_{k}\right)
\end{array}\right)=\left(\begin{array}{c}
2^{n-1} \\
\ldots \\
2^{n-1}
\end{array}\right)\right)=\mathbf{P}\left(\sum_{\alpha \in V_{n-k}} \eta_{\alpha}=\left(\begin{array}{c}
2^{n-2} \\
\ldots \\
2^{n-2}
\end{array}\right)\right) .
\end{gathered}
$$

Лемма 1. Справедливо равенство

$$
M \eta_{\alpha}^{i} \eta_{\alpha}^{j}=2^{2(k-2)}+2^{k-3} I\{i=j\}, \quad 1 \leqslant i, j \leqslant k .
$$

Доказательство. Справедливо равенство

$$
\eta_{\alpha}^{i}=\frac{1}{2} \sum_{t \in V_{k}}\left(f(t \alpha) \oplus f\left(t \alpha \oplus e_{i}\right)\right)
$$

Если $i=j$, то $\mathrm{M}\left(\eta_{\alpha}^{i}\right)^{2}$ - второй момент случайной величины с биномиальным распределением с параметрами $\left(2^{k-1}, 1 / 2\right)$ (суммы $2^{k-1}$ независимых равновероятных индикаторов). 
Если $i \neq j$, то

$$
\mathbf{M} \eta_{\alpha}^{i} \eta_{\alpha}^{j}=\frac{1}{4} M \sum_{t_{1}, t_{2} \in V_{k}}\left(f\left(t_{1} \alpha\right) \oplus f\left(t_{1} \alpha \oplus e_{i}\right)\right)\left(f\left(t_{2} \alpha\right) \oplus f\left(t_{2} \alpha \oplus e_{j}\right)\right)
$$

Значения $f$ в разных точках независимы и равновероятно распределены на $V=\{0,1\}$. При любых $t_{1}, t_{2}$ и $i \neq j$, каждый сомножитель содержит слагаемое, не зависящее от второго, следовательно, сомножители независимы.

Лемма 1 доказана.

Продолжим доказательство теоремы. Из равенств (3) следует, что $\mathrm{D} \eta_{\alpha}^{i}=2^{k-3}$ и $\operatorname{cov} \eta_{\alpha}^{i} \eta_{\alpha}^{j}=0$ при $i \neq j$.

Следовательно, ковариационная матрица случайного вектора $\eta_{\alpha}$ имеет вид $\operatorname{Cov} \eta_{\alpha}=2^{k-3} I$, где $I$ - единица в кольце квадратных матриц размера $k$.

Рассмотрим решетку $Z_{k}$ всех целочисленных векторов размерности $k$ - дискретную подгрупाту в множестве всех вещественных векторов размерности $k$. Ясно, что

$$
\mathbf{P}\left(\eta_{\alpha} \in Z_{k}\right)=1 .
$$

Следовательно, $\eta_{\alpha}-$ решетчатый случайный вектор.

Согласно [7] решетка $M$ называется минимальной для случайного вектора $X$, если она удовлетворяет следующим двум условиям:

(1) $\mathbf{P}(X \in x+M)=1$ для любого $x$ такого, что $\mathbf{P}(X=x)>0$,

(2) если существует подгруппа $C$ множества всех вещественных векторов размерности $k$ такая, что $\mathbf{P}(X \in y+C)=1$ при некотором $y$, то $M \subseteq C$.

Пусть

$$
\bar{e}=\sum_{j=1}^{k} e_{j}, \quad e_{i} \in V_{k}, \quad i=1, \ldots, k
$$

Рассмотрим множество $L$ такое, что

$$
L=2 Z_{k} \cup\left\{2 Z_{k}+\bar{e}\right\} .
$$

Лемма 2. Множество L является минимальной решеткой для случайного вектора $\eta_{\alpha}$.

Доказательство. Очевидно, что $L$ является решеткой.

Рассмотрим распределение случайного вектора $\eta_{\alpha}$. Многочлен Жегалкина функции $f$ можно единственным образом представить в виде

$$
f\left(x_{1}, \ldots, x_{n}\right)=\bigoplus_{\nu \in V_{k}} x^{\nu} f_{\nu}\left(x_{k+1}, \ldots, x_{n}\right),
$$

где $x=\left(x_{1}, \ldots, x_{k}\right), v=\left(v_{1}, \ldots, v_{k}\right), x^{\nu}=x_{1}^{\nu_{1}} \ldots x_{k}^{\nu_{k}}$, а знак $\bigoplus$ означает сложение по модулю 2.

Случайному и равновероятному выбору $f$ из множества $B_{n}$ соответствует случайный, независимый и равновероятный выбор из множества $B_{n-k}$ набора из $2^{k}$ функций $f_{v}$, $v \in V_{k}$, значения которых можно рассматривать как независимые случайные величины, равномерно распределенные на $V$. 
Несложно убедиться, что случайную величину $\eta_{\alpha}^{i}$ можно представить в виде

$$
\eta_{\alpha}^{i}=\sum_{m=0}^{k-1} \sum_{J \subset\{1, \ldots, k\} \backslash\{i\}} \bigoplus_{\mu \in V_{m}} f_{e_{i} \oplus \mu_{1} e_{j_{1}}} \oplus \ldots \oplus \mu_{m} e_{j_{m}}(\alpha),
$$

где $|J|=m, J=\left\{j_{1}, \ldots, j_{m}\right\}, \mu=\left(\mu_{1}, \ldots, \mu_{m}\right)$.

Если в представлении $\eta_{\alpha}^{i}$ перейти от суммирования по модулю 2 к обычному суммированию в поле действительных чисел, использовав то, что

$$
f_{1}(\alpha) \oplus f_{2}(\alpha)=\left(f_{1}(\alpha)-f_{2}(\alpha)\right)^{2}=f_{1}(\alpha)+f_{2}(\alpha)-2 f_{1}(\alpha) f_{2}(\alpha)
$$

нетрудно увидеть, что

$$
\eta_{\alpha}=\left(\begin{array}{c}
2 G_{1}\left(f_{(1 t)}(\alpha), t \in V_{k-1}, t \neq \bar{e}\right)+f_{(11 \ldots 11)}(\alpha) \\
\ldots \\
\left.2 G_{1}\left(f_{\left(t_{1} \ldots t_{i-1} 1\right.} t_{i} \ldots t_{k-1}\right)(\alpha), t=\left(t_{1} \ldots t_{k-1}\right) \in V_{k-1}, t \neq \bar{e}\right)+f_{(11 \ldots 11)}(\alpha) \\
\ldots \\
\left.2 G_{k}\left(f_{(t 1)}(\alpha), t \in V_{k-1}, t \neq \bar{e}\right)+f_{(11 \ldots 11}\right)(\alpha)
\end{array}\right)
$$

где $G_{1}\left(x_{i}, i \in\left\{1,2^{k-1}-1\right\}\right), \ldots, G_{k}\left(x_{i}, i \in\left\{1,2^{k-1}-1\right\}\right)$ - некоторые многочлены от $2^{k-1}-1$ переменных. Следовательно, случайный вектор $\eta_{\alpha}$ принимает значения либо из множества $\left\{2 Z_{k}\right\}$, либо из множества $\left\{2 Z_{k}+\bar{e}\right\}$. Получаем, что $\mathbf{P}\left(\eta_{\alpha} \in L\right)=1$.

Нетрудно убедиться, что базисом $L$ является набор векторов вида

$$
\left\{2 e_{1}, 2 e_{2}, \ldots, 2 e_{k-1}, \bar{e}\right\} \text {. }
$$

Если случайная величина $f_{\bar{e} \oplus e_{i}}(\alpha)$ принимает значение 1 , а $f_{v}$ принимает значение 0 для прочих $v \in V_{k}$, то нетрудно убедиться, что $\eta_{\alpha}$ принимает значение $2 \bar{e}-2 e_{i}$.

Аналогично, если $f_{\bar{e}}(\alpha)=1$, а $f_{\nu}=0$ для прочих $v \in V_{k}$, то $\eta_{\alpha}$ принимает значение $\bar{e}$. Для любого $i, 0<i<k$, справедливо равенство $2 e_{i}=2 \bar{e}-\left(2 \bar{e}-2 e_{i}\right)$, следовательно, можно сделать вывод, что если $\mathbf{P}\left(\eta_{\alpha} \in M\right)=1$, где $M$ - некоторая подгруппа множества всех вещественных векторов размерности $k$, то $L \subseteq M$. Следовательно, $L-$ минимальная решетка для $\eta_{\alpha}$.

Лемма 2 доказана.

Вернемся к доказательству теоремы и рассмотрим случайный вектор $X_{\alpha}=T \eta_{\alpha}$, где $T=2^{-(k-3) / 2} I$.

Очевидно, что

$$
\mathbf{M} X_{\alpha}=2^{(k-1) / 2} \bar{e}, \quad \operatorname{Cov} X_{\alpha}=I .
$$

Нетрудно убедиться, что $\mathbf{M}\left\|X_{\alpha}-\mathbf{M} X_{\alpha}\right\|^{3}<\infty$.

Повторив доказательство леммы 2 с точностью до умножения на матрицу $T$, можно заметить, что минимальная решетка $L^{\prime}$ для случайного вектора $X_{\alpha}$ имеет базис

$$
\left\{2^{(5-k) / 2} e_{1}, \ldots, 2^{(5-k) / 2} e_{k-1}, 2^{(3-k) / 2} \bar{e}\right\},
$$

и следовательно, модуль определителя матрицы, составленной из базисных векторов (определитель решетки, не зависящий от выбора базиса), равен

$$
\operatorname{det} L^{\prime}=2^{-\left(k^{2}-5 k+2\right) / 2} \text {, }
$$


и

$$
\begin{aligned}
\mathbf{P}\left(\sum_{\alpha \in V_{n-k}} \eta_{\alpha}=\left(\begin{array}{c}
2^{n-2} \\
\ldots \\
2^{n-2}
\end{array}\right)\right) & =\mathbf{P}\left(\sum_{\alpha \in V_{n-k}} X_{\alpha}=T\left(\begin{array}{c}
2^{n-1} \\
\ldots \\
2^{n-1}
\end{array}\right)\right) \\
& =\mathbf{P}\left(\sum_{\alpha \in V_{n-k}} X_{\alpha}=\left(\begin{array}{c}
2^{(2 n-k-1) / 2} \\
\ldots \\
2^{(2 n-k-1) / 2}
\end{array}\right)\right) .
\end{aligned}
$$

Таким образом, при $n \rightarrow \infty$ для суммы $2^{n-k}$ независимых одинаково распределенных случайных векторов $X_{\alpha}$ выполнены условия локальной предельной теоремы из [7] для сумм независимых одинаково распределенных случайных векторов и, следовательно,

$$
\begin{aligned}
\left(1+\left\|y_{\theta, 2^{n-k}}\right\|^{3}\right) \mathbf{P} & \left(\sum_{\alpha \in V_{n-k}} X_{\alpha}=\theta\right)=\frac{1}{2^{(n-k) k / 2}(2 \pi)^{-k / 2}} \operatorname{det} L^{\prime} \exp \left(-\frac{1}{2}\left\|y_{\theta, 2^{n-k}}\right\|^{2}\right) \\
& +\frac{1}{2^{(n-k)(k+1) / 2}(2 \pi)^{-k / 2}} \operatorname{det} L^{\prime} \exp \left(-\frac{1}{2}\left\|y_{\theta, 2^{n-k}}\right\|^{2}\right) \\
& \times\left\{-\frac{1}{6}\left(\chi_{(3,0, \ldots, 0)}\left(-y_{1}^{3}+3 y_{1}\right)+\ldots+\chi_{(0,0, \ldots, 3)}\left(-y_{k}^{3}+3 y_{k}\right)\right)\right. \\
& -\frac{1}{2}\left(\chi_{(2,1, \ldots, 0)}\left(-y_{1}^{2} y_{2}+y_{2}\right)+\ldots+\chi_{(0, \ldots, 1,2)}\left(-y_{k}^{2} y_{k-1}+y_{k-1}\right)\right) \\
& \left.-\chi_{(1,1,1,0, \ldots, 0)}\left(-y_{1} y_{2} y_{3}\right)+\chi_{(0, \ldots, 1,1,1)}\left(-y_{k-2} y_{k-1} y_{k}\right)\right\}+o\left(2^{-n(k+1) / 2}\right),
\end{aligned}
$$

где $y_{\theta, 2^{n-k}}=\left(y_{1}, \ldots, y_{k}\right)=2^{-(n-k) / 2}\left(\theta-2^{n-k} M X_{\alpha}\right)$, а $\chi_{\left(\gamma_{1}, \ldots \gamma_{k}\right)}=\chi_{\gamma}-$ семиинвариант случайного вектора $X_{\alpha}$ порядка $\gamma$.

В нашем случае

$$
\theta=2^{(2 n-k-1) / 2} \bar{e}, \quad y_{\theta, 2^{n-k}}=2^{-(n-k) / 2}\left(2^{(2 n-k-1) / 2} \bar{e}-2^{n-k} 2^{(k-1) / 2} \bar{e}\right)=\overline{0},
$$

где $\overline{0} \in V_{k}$ - вектор веса 0 , следовательно,

$$
\begin{aligned}
\mathbf{P}\left(\sum_{\alpha \in V_{n-k}} X_{\alpha}\right. & \left.=\left(\begin{array}{c}
2^{(2 n-k-1) / 2} \\
\ldots \\
2^{(2 n-k-1) / 2}
\end{array}\right)\right) \\
& =2^{-\left(k^{2}-5 k+2\right) / 2} 2^{-(n-k) k / 2}(2 \pi)^{-k / 2}+o\left(2^{-n(k+1) / 2}\right), \\
\frac{1}{2^{2^{n}}} S(n, k) & =\mathbf{P}\left(\sum_{\beta \in V_{n}}\left(\begin{array}{c}
f(\beta) \oplus f\left(\beta \oplus e_{1}\right) \\
f(\beta) \oplus f\left(\beta \oplus e_{k}\right)
\end{array}\right)=\left(\begin{array}{c}
2^{n-1} \\
\ldots \\
2^{n-1}
\end{array}\right)\right) \\
& =\mathbf{P}\left(\sum_{\alpha \in V_{n-k}} \eta_{\alpha}=\left(\begin{array}{c}
2^{n-2} \\
\ldots \\
2^{n-2}
\end{array}\right)\right)=\mathbf{P}\left(\sum_{\alpha \in V_{n-k}} X_{\alpha}=\left(\begin{array}{c}
2^{(2 n-k-1) / 2} \\
\ldots \\
2^{(2 n-k-1) / 2}
\end{array}\right)\right) \\
& =2^{2 k-1} \pi^{-k / 2} 2^{-n k / 2}+o\left(2^{-n(k+1) / 2}\right) .
\end{aligned}
$$

Теорема 1 доказана.

Данная теорема при $k=1$ предлагает асимптотическую формулу для $S(n, 1)$, совпадающую с формулой, доказанной в [2]. 
Следствие 1. Пусть $k$ - некоторое натуральное число, $\varepsilon$ - положительное вещественное число. Тогда существует натуральное число $N$ такое, что для любых $n>N$

$$
S^{n}<(1+\varepsilon) \pi^{-k / 2} 2^{2^{n}-n k / 2+2 k-1} .
$$

Доказательство. Воспользуемся неравенством $S^{n}<S(n, k)$. Согласно теореме 1

$$
\lim _{n \rightarrow \infty} S(n, k) / 2^{2^{n}} 2^{2 k-1} \pi^{-k / 2} 2^{-n k / 2}=1 .
$$

Следовательно, для любого $\varepsilon>0$ найдется $N$ такое, что для всех $n>N$

$$
S(n, k)<(1+\varepsilon) 2^{2 k-1} \pi^{-k / 2} 2^{2^{n}-n k / 2} .
$$

\section{Следствие 1 доказано.}

Теперь, используя результаты данной работы и работы [5], можно выписать следующую двустороннюю оценку для $S^{n}$ при произвольном положительном $\varepsilon$ и фиксированном $k$ при всех достаточно больших $n$ :

$$
\frac{\exp _{2}\left(2^{n}-n^{2} / 2+n\right)}{\pi^{n / 2}} \leqslant S^{n}<\frac{(1+\varepsilon) 2^{2 k-1} \exp \left(2^{n}-n k / 2\right)}{\pi^{k / 2}} .
$$

\section{Список литературы}

1. Webster A. F., Tavares S. E., On the design of $S$-boxes. Lecture Notes Comput. Sci. (1986) 218 , 523-534.

2. Cusick T. W., Bounds on the number of functions satisfying the strict avalanche criterion. Inform. Processing Lett. (1996) 57, 261-263.

3. Cusick T. W., Stanica P., Bounds on the number of functions satisfying the strict avalanche criterion. Inform. Processing Lett. (1996) 60, 215-219.

4. Youssef A. M., Tavares S. E., Comment on bounds on the number of functions satisfying the strict avalanche criterion. Inform. Processing Lett. (1996) 60, 271-275.

5. Bliss D. K., A lower bound on the number of functions satisfying the strict avalanche criterion. Discrete Math. (1998) 185, 29-39.

6. O'Connor L., An upper bound on the number of functions satisfying the strict avalanche criterion. Inform. Processing Lett. (1994) 52, 325-327.

7. Бхаттачария Р. Н., Ранга Рао Р., Аппроксимачия нормальным распределением и асимптотические разложения. Наука, Москва, 1982.

Статья поступила 05.10.2004. 